\title{
Feasibility of tele-ophthalmology for screening for eye disease in remote communities
}

\author{
Li-Sheng Chen*, Ching-Yao Tsai , Tzeng-Ying Lius, Tao-Hsin Tung , \\ Yueh-Hsia Chiu*, Chang-Chuan Chan**, Der-Ming Liou* and \\ Tony Hsiu-Hsi Chen
}

*Institute of Public Health and Institute of Health Informatics and Decision Making, School of Medicine, National Yang-Ming University, Taipei; 'Department of Ophthalmology, Taipei Municipal Chung-Hsin Hospital; Institute of Public Health, School of Medicine, National Yang-Ming University, Taipei; §Health Bureau of Lienkiang County, Matsu; **Institute of Occupational Medicine and Industrial Hygiene, College of Public Health, National Taiwan University; ${ }^{\dagger \dagger}$ Institute of Preventive Medicine, College of Public Health, National Taiwan University, Taipei, Taiwan

\begin{abstract}
Summary
We assessed the feasibility of tele-ophthalmology in a remote location, Tungyin, an island $200 \mathrm{~km}$ from Taiwan, which has no ophthalmologist. Screening for eye diseases was carried out among residents aged 40 years or more. A total of 113 subjects, approximately $31 \%$ of the whole population, were enrolled in the screening programme. Images were transmitted (via ADSL) to a retinal specialist in Taiwan for diagnosis. The average processing time, excluding the time for copying files, was $6.4 \mathrm{~s}$ (SD 2.1) per subject. Transmission took $60-90$ s for most of the images (83\%). The average time required to make a diagnosis for each subject, including data entry, was approximately $34 \mathrm{~s}$ (SD 18). In screening for retinopathy, the detection rate with digital imaging (8.8\%) was two times higher than with indirect ophthalmoscopy (4.4\%). In $12 \%$ of cases macular degeneration was identified, and in $6 \%$ there were mild or moderate problems with the optic disc. Community-based screening for four categories of eye disease was successfully demonstrated using store-andforward tele-ophthalmology.
\end{abstract}

\section{Introduction}

Tele-ophthalmology may be useful in remote areas where there are difficulties in accessing an ophthalmologist. For example, acute ophthalmological conditions in an Australian rural emergency department have been managed by teleconsultation with ophthalmologists working in a specialist eye clinic $900 \mathrm{~km}$ away ${ }^{1}$. A pilot astigmatism screening study evaluated the transmission of digital images of children's pupil light reflexes to an ophthalmologist for diagnosis ${ }^{2}$. Studies have tested the feasibility of teleophthalmology in the examination of patients with

Accepted 5 September 2004

Correspondence: Professor Tony Hsiu-Hsi Chen, Graduate Institute of Preventive Medicine, College of Public Health, National Taiwan University, Room 207, 19 Hsuchow Road, Taipei, Taiwan (Fax: +886 22358 7707; Email: stony@episerv.cph.ntu.edu.tw) glaucoma ${ }^{3-5}$ as well as for diabetic retinopathy screening $^{6-9}$ (though not all of the latter found that it was feasible). Some studies have demonstrated the feasibility of using a portable fundus camera and a digital indirect ophthalmoscope for screening for common blinding eye diseases ${ }^{10,11}$.

Despite this body of work, there is still a need to assess the feasibility of tele-ophthalmology for population-based screening for retinopathy and certain other eye diseases in remote areas, which typically have fewer than one ophthalmologist per 100,000 population. According to Lamminen et al. ${ }^{12}$ the success of implementing tele-ophthalmology depends on technical aspects (e.g. appropriate facilities, the sending and reading of digital messages) as well as organizational and operational factors.

We have therefore investigated whether teleophthalmology is feasible for community-based 
screening for retinopathy and other eye diseases in a remote area that has no ophthalmologist.

\section{Methods}

For the present study we selected a remote location, Tungyin, one of the Matsu islets, which is $33 \mathrm{~km}$ from mainland China and $200 \mathrm{~km}$ from Taiwan (Fig 1). In Tungyin there are many elderly people who require screening for retinopathy and other eye diseases, including macular oedema, optic disc problems and glaucoma. However, there is no ophthalmologist. There is ADSL communication between Taiwan and Tungyin. A tele-ophthalmology community screening programme for these four selected eye problems was tested.

\section{Study population}

There were 367 residents aged 40 years or more in Tungyin. The study population (i.e. those enrolled to attend the screening programme) comprised 113 residents, or approximately $31 \%$ of the target population. Their mean age was 53 years (SD 13). The most common reason for being unable to attend the screening programme among the 254 non-attenders was that they were not in Tungyin at the time, but were travelling (e.g. to visit relatives).

Only residents aged 40 years or older were invited to participate, but 14 residents aged 30-39 years also attended.

\section{Study design and clinical assessment}

The study design varied across the four different categories of eye disease of interest: retinopathy, macular oedema, optic disc problems and glaucoma. For the detection of retinopathy, we used both indirect ophthalmoscopy (one of the established tools for

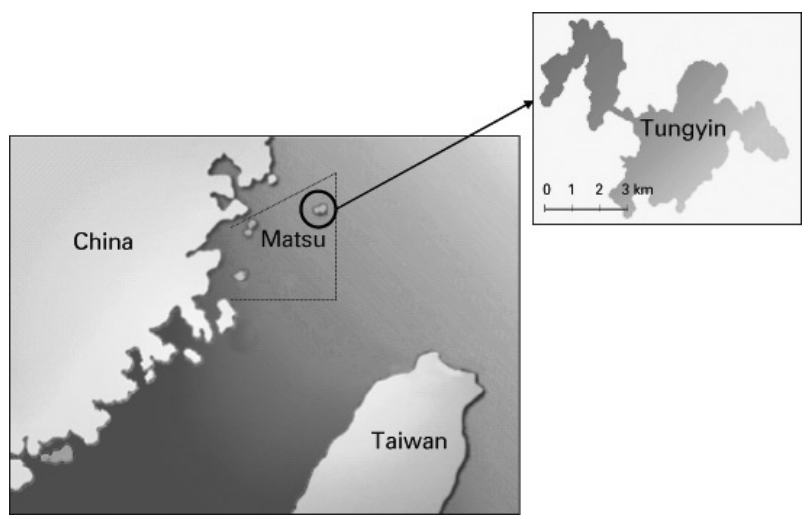

Fig 1 Locations of Tungyin in the Matsu islands and Taiwan. diabetic retinopathy screening) and digital imaging. We compared detection rates between digital imaging and indirect ophthalmoscopy. The detection of macular oedema and optic disc problems was entirely based on digital imaging. The diagnosis of glaucoma was made according to the anatomical findings from the patient's optic nerve disc, and functional visual field examination by frequency-doubling perimetry (FDP). Intraocular pressure (IOP) was also evaluated. An elevated IOP was defined as over $17 \mathrm{mmHg}$ $(1 \mathrm{mmHg}=133 \mathrm{~Pa})$. Severe glaucoma was defined as an optic cup:disc ratio over 0.7 with an FDP defect or elevated IOP. Mild glaucoma was defined as an optic cup:disc ratio between 0.7 and 0.5 , or disc asymmetry of over 20\%, with an FDP defect or elevated IOP.

The equipment and measures used in the clinical assessments included objective auto-refraction, measurements of presenting and best-corrected visual acuity, measurement of IOP with a non-contact tonometer, measurements of ocular dimensions, automated FDP, external and anterior segment examinations with a slit-lamp biomicroscope, and examinations of the vitreous, retina and optic nerve with an indirect ophthalmoscope. Digital 35-degree colour fundus images were obtained with a nonmydriatic digital fundus camera (CR6-45, Canon, Japan).

\section{Tele-ophthalmology procedure}

The examination procedure is shown in Fig 2. Images of each fundus were checked using the non-mydriatic

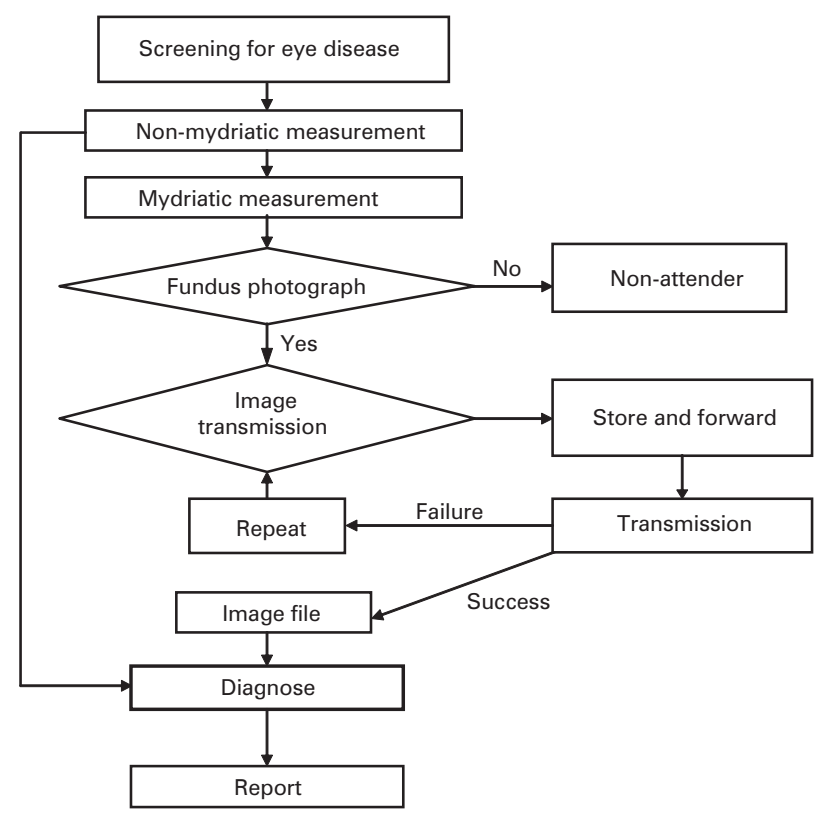

Fig 2 Procedure for the tele-ophthalmology screening programme. 
digital fundus imaging system with two-field images. Images were transmitted to a retinal specialist in Taiwan for diagnosis. The retinal specialist reviewed each image, made any relevant diagnosis and made recommendations for subsequent care.

Each image was $2048 \times 1360$ pixels, at a colour depth of $24 \mathrm{bit} /$ pixel. As previous studies had shown that JPEG compression of 1:28 for colour images was acceptable, fundus photographs were compressed 1:20 before transmission ${ }^{13}$. Transmission was by ADSL. The lowest upload speed was $64 \mathrm{kbit} / \mathrm{s}$ and the download speed was $512 \mathrm{kbit} / \mathrm{s}$.

\section{Results}

\section{Time required to process the image files}

The first step was to transfer retinal images from the digital camera and to name each file appropriately. Two assistants compressed the image files. The average processing time, excluding the time for copying files, was $6.4 \mathrm{~s}$ (SD 2.1) per subject (Table 1).

\section{Time required for image transmission}

Assistants started to transmit the files using batch processing after every 20-30 subjects. Because of problems with the ADSL link in the remote area, it was

Table 1 Time required for renaming and compressing image files

\begin{tabular}{lccl}
\hline Session number & $\begin{array}{l}\text { Number of } \\
\text { subjects }\end{array}$ & Time (s) & $\begin{array}{l}\text { Mean time } \\
\text { per subject (s) }\end{array}$ \\
\hline 1 & 24 & 180 & 7.5 \\
2 & 32 & 240 & 7.5 \\
3 & 7 & 60 & 8.6 \\
4 & 32 & 180 & 5.6 \\
5 & 18 & 60 & 3.3 \\
Total & 113 & 720 & 6.4 \\
\hline
\end{tabular}

sometimes necessary to attempt to transmit the images up to nine times. Table 2 shows the number of attempts and time required for the successful transmission of the image files. The majority of image files $(88 \%)$ required five or fewer attempts at transmission. For most of the subjects $(83 \%)$, transmission took 60-90 s (Table 2).

\section{Time required for image diagnosis}

The retinal specialists used a Web browser to view the digital images. They had been previously trained to use the application software, which was easy to use (it is available upon request from the first author). For diagnosis, each image could be zoomed in or out for further viewing. The average diagnosis time for each subject, including data entry, was approximately $34 \mathrm{~s}$ (SD 18).

\section{Results of screening}

Table 3 shows the retinopathy results for all 113 subjects. Retinopathy was graded according to a modified Airlie House Classification System ${ }^{14}$. Digital imaging detected 10 cases of 'other retinopathy' (six cases in the right eye and four cases in the left). By comparison, indirect ophthalmoscopy detected only five cases of 'other retinopathy' (three in the right eye, two in the left). No diabetic retinopathy was detected. Images from three subjects were undiagnosable due to failure of the digital fundus imaging system. In screening for retinopathy, the detection rate for digital imaging (8.8\%) was two times higher than with indirect ophthalmoscopy (4.4\%).

The screening results for the other three eye problems are summarized in Table 4. Among the 113 participants, we found 14 cases of early age-related macular degeneration (AMD) and found 14 other cases of AMD. In the optic disc assessment, we found six cases with mild problems and eight cases with moderate problems. There were no severe problems.

Table 2 Number of transmission attempts and time required

\begin{tabular}{|c|c|c|c|c|c|c|c|}
\hline \multirow[b]{2}{*}{ Number of transmission attempts required } & \multicolumn{7}{|c|}{ Transmission time for image (s) } \\
\hline & $0-59$ & $60-89$ & 90-119 & $120-179$ & $180-239$ & $240+$ & Total \\
\hline 1 & 0 & 8 & 2 & 2 & 0 & 0 & 12 \\
\hline 2 & 0 & 13 & 2 & 6 & 1 & 0 & 22 \\
\hline 3 & 0 & 8 & 1 & 0 & 0 & 1 & 10 \\
\hline 4 & 1 & 27 & 0 & 0 & 0 & 0 & 28 \\
\hline 5 & 1 & 25 & 1 & 0 & 0 & 0 & 27 \\
\hline 6 & 0 & 5 & 0 & 0 & 0 & 0 & 5 \\
\hline 7 & 0 & 3 & 0 & 0 & 0 & 0 & 3 \\
\hline 8 & 1 & 1 & 0 & 0 & 0 & 0 & 2 \\
\hline 9 & 0 & 4 & 0 & 0 & 0 & 0 & 4 \\
\hline Total & $3(3 \%)$ & 94 (83\%) & $6(5 \%)$ & $8(7 \%)$ & 1 (1\%) & 1 (1\%) & 113 \\
\hline
\end{tabular}


Table 3 Grade of retinopathy detected with retinal digital photography and indirect ophthalmoscopy

\begin{tabular}{|c|c|c|c|c|c|c|c|c|}
\hline \multirow[b]{3}{*}{ Retinopathy } & \multicolumn{4}{|c|}{ Right eye } & \multicolumn{4}{|c|}{ Left eye } \\
\hline & \multicolumn{2}{|c|}{ Digital imaging } & \multicolumn{2}{|c|}{ Indirect ophthalmoscopy } & \multicolumn{2}{|c|}{ Digital imaging } & \multicolumn{2}{|c|}{ Indirect ophthalmoscopy } \\
\hline & $n$ & $\%$ & $n$ & $\%$ & $n$ & $\%$ & $n$ & $\%$ \\
\hline None & 104 & 92 & 110 & 97 & 109 & 96 & 111 & 98 \\
\hline NPDR & 0 & 0 & 0 & 0 & 0 & 0 & 0 & 0 \\
\hline Mild NPDR & 0 & 0 & 0 & 0 & 0 & 0 & 0 & 0 \\
\hline Severe NPDR & 0 & 0 & 0 & 0 & 0 & 0 & 0 & 0 \\
\hline PDR & 0 & 0 & 0 & 0 & 0 & 0 & 0 & 0 \\
\hline Severe PDR & 0 & 0 & 0 & 0 & 0 & 0 & 0 & 0 \\
\hline Other retinopathy & 6 & 5 & 3 & 3 & 4 & 4 & 2 & 2 \\
\hline Insufficient image quality for diagnosis & 3 & 3 & 0 & 0 & 0 & 0 & 0 & 0 \\
\hline Total & 113 & 100 & 113 & 100 & 113 & 100 & 113 & 100 \\
\hline
\end{tabular}

NPDR, non-proliferative diabetic retinopathy.

PDR, proliferative diabetic retinopathy.

Table 4 Screening results for macular degeneration, optic disc problems and suspected glaucoma

\begin{tabular}{|c|c|c|c|c|}
\hline \multirow[b]{2}{*}{ Eye disease } & \multicolumn{2}{|c|}{ Right eye } & \multicolumn{2}{|c|}{ Left eye } \\
\hline & $n$ & $\%$ & $n$ & $\%$ \\
\hline \multicolumn{5}{|l|}{ Macular degeneration } \\
\hline None & 102 & 90 & 96 & 85 \\
\hline Early age-related macular degeneration & 6 & 5.3 & 8 & 7.1 \\
\hline Macular degeneration & 5 & 4.4 & 9 & 8.0 \\
\hline \multicolumn{5}{|l|}{ Optic disc assessment } \\
\hline Normal & 105 & 93 & 107 & 95 \\
\hline Mild problems & 3 & 2.7 & 3 & 2.7 \\
\hline Moderate problems & 5 & 4.4 & 3 & 2.7 \\
\hline Severe problems & 0 & 0 & 0 & 0 \\
\hline \multicolumn{5}{|l|}{ Suspect glaucoma } \\
\hline None & 96 & 85 & 96 & 85 \\
\hline Mild glaucoma & 1 & 0.9 & 4 & 3.5 \\
\hline Severe glaucoma & 2 & 1.8 & 2 & 1.8 \\
\hline High intraocular pressure & 14 & 12.4 & 11 & 9.7 \\
\hline Total & 113 & 100 & 113 & 100 \\
\hline
\end{tabular}

The results of the comprehensive glaucoma assessment (FDP, IOP and fundus images) are also summarized in Table 4 . We found five cases of mild glaucoma and four cases of severe glaucoma. High IOP ( $>17 \mathrm{mmHg}$ ) (a risk factor for glaucoma) was found in 25 cases.

\section{Discussion}

Transmission methods in telemedicine can be grouped into three broad categories: store and forward, realtime and hybrid ${ }^{13}$. Store-and-forward systems relay data asynchronously. Information is acquired at one site, stored on a computer and then transmitted at a later time to another location, where it may be stored again before review. Realtime systems work synchronously. Speakers, microphones and television cameras allow live videoconferences and group whiteboards to pass information almost instantaneously. Hybrid systems combine the capabilities of realtime and store-andforward telemedicine ${ }^{13}$. Our study used store-andforward methods to transmit image files because of their flexibility compared with realtime transmission. Although we had ADSL communication available between Taiwan and Tungyin, this was not always stable. Except when retransmission was required, the time required for renaming and compressing image files, the time required for image transmission, and the time required for diagnosis totalled approximately 2 minutes.

In the community screening study, no diabetic retinopathy was detected. This does not mean that our tele-ophthalmology method is inadequate for this purpose. The explanation is that because our target population was based on a general population, the prevalence diabetic retinopathy was low.

The results suggest that tele-ophthalmology can detect early age-related macular oedema (12\% of the study population), optic disc problems (mild and moderate in a total of $12 \%$ of the study population) and high IOP (22\%). Early treatment of these eye diseases can reduce the chance of blindness. However, the accuracy of detection through digital imaging requires further validation.

Two characteristics of the tele-ophthalmology screening programme deserve mention. First, our teleophthalmology was conducted from a mobile unit that moved between screening sites. Second, the target population was not patients who had sought medical service due to the presence of signs or symptoms but, unlike in earlier studies, was based on residents who participated annually in a multiple screening programme. This facilitated the early detection of eye disease. 
Our study is not novel in the realm of telemedicine. However, the feasibility of a community-based teleophthalmology screening programme for the early detection of eye disease was demonstrated. It would be useful to know the sensitivity and specificity of the technique in the detection of posterior eye conditions. However, this would require all patients with negative findings to be sent to hospital for confirmatory diagnosis.

Cost is also an important factor in a screening programme. Our estimated cost per patient was about US\$10 (€8), which was slightly lower than that estimated in a study by Leese et al. ${ }^{15}$, who also used a mobile screening unit for diabetic retinopathy, in the UK. As our screening covered more than diabetic retinopathy, this suggests that our tele-ophthalmology was efficient.

Third, as suggested by Lamminen et al. ${ }^{12}$, the quality of tele-ophthalmology is also important. Concerns have been raised as to whether lower image quality due to compression may affect diagnosis. In the present study this was unlikely, for two reasons. First, previous studies have shown that JPEG compression of 1:28 is not apparent to observers, and we chose a lower degree of compression, 1:20. Second, the digital imaging method had a higher detection rate for retinopathy than indirect ophthalmoscopy (see Table 3).

In conclusion, the community-based teleophthalmology screening programme was successful in remote Tungyin, Taiwan. The system was useful for the early detection of retinopathy, macular oedema, optic disc problems and glaucoma. Early treatment of these may reduce the incidence of blindness.

\section{References}

1 Rosengren D, Blackwell N, Kelly G, Lenton L, Glastonbury J. The use of telemedicine to treat ophthalmological emergencies in rural Australia. Journal of Telemedicine and Telecare 1998;4 (suppl. 1):97-9

2 Researchers to test teleophthalmology on the reservation. Telemedicine and Virtual Reality 1997;2:121, 131

3 Yogesan K, Constable IJ, Eikelboom RH, van Saarloos PP. Teleophthalmic screening using digital imaging devices. Australian and New Zealand Journal of Ophthalmology 1998;26 (suppl. 1):9-11

4 Tuulonen A, Ohinmaa T, Alanko HI, Hyytinen P, Juutinen A, Toppinen E. The application of teleophthalmology in examining patients with glaucoma: a pilot study. Journal of Glaucoma 1999; 8:367-73

5 Lamminen H. Picture archiving and fundus imaging in a glaucoma clinic. Journal of Telemedicine and Telecare 2003;9:114-16

6 Williamson TH, Keating D. Telemedicine and computers in diabetic retinopathy screening. British Journal of Ophthalmology 1998;82:5-6

7 Schwartz SD, Harrison SA, Ferrone PJ, Trese MT. Telemedical evaluation and management of retinopathy of prematurity using a fiberoptic digital fundus camera. Ophthalmology 2000;107:25-8

8 Gomez-Ulla F, Fernandez MI, Gonzalez F, et al. Digital retinal images and teleophthalmology for detecting and grading diabetic retinopathy. Diabetic Care 2002;25:1384-9

9 Rotvold GH, Knarvik U, Johansen MA, Fossen K. Telemedicine screening for diabetic retinopathy: staff and patient satisfaction. Journal of Telemedicine and Telecare 2003;9:109-13

10 Schiffman JS, Li HK, Tang RA. Telemedicine enters eye care: practical experience. Journal of Ophthalmic Nursing and Technology 1998;17:102-6

11 Constable IJ, Yogesan K, Eikelboom R, Barry C, Cuypers M. Fred Hollows lecture: digital screening for eye disease. Clinical and Experimental Ophthalmology 2000;28:129-32

12 Lamminen H, Voipio V, Ruohonen K, Uusitalo H. Telemedicine in ophthalmology. Acta Ophthalmologica Scandinavica 2003;81:105-9

13 Li HK. Telemedicine and ophthalmology. Survey of Ophthalmology 1999;44:61-72

14 Klein R, Klein BE, Magli YL, et al. An alternative method of grading diabetic retinopathy. Ophthalmology 1986;93:1183-7

15 Leese GP, Ahmed S, Newton RW, et al. Use of mobile screening unit for diabetic retinopathy in rural and urban areas. British Medical Journal 1993;306:187-9 